\title{
Variabilidade Espaço-Temporal das Tendências de Precipitação na Mesorregião Sul Cearense e sua Relação com as Anomalias de TSM
}

\author{
Pedro dos Santos Ferreira ${ }^{1}$, Werônica Meira de Souza ${ }^{2}$, Jadson Freire da Silva ${ }^{3}$ iD , \\ Viviane Pedroso Gomes ${ }^{4}$ \\ ${ }^{1}$ Programa de Pós-Graduação em Desenvolvimento e Meio Ambiente, \\ Universidade Federal de Pernambuco, Recife, PE, Brasil. \\ ${ }^{2}$ Unidade Acadêmica de Garanhuns, Universidade Federal Rural de Pernambuco, \\ Garanhuns, PE, Brasil. \\ ${ }^{3}$ Programa de Pós-Graduação em Desenvolvimento e Meio Ambiente, \\ Universidade Federal de Pernambuco, Recife, PE, Brasil. \\ ${ }^{4}$ Programa de Pós-Graduação em Desenvolvimento e Meio Ambiente, \\ Universidade Federal de Pernambuco, Recife, PE, Brasil.
}

Recebido em 14 de Maio de 2017 - Aceito em 29 de Setembro de 2017

\begin{abstract}
Resumo
A preocupação com as mudanças climáticas tem suscitado uma série de questões relacionadas aos extremos de precipitação no semiárido brasileiro, cada vez mais discutidas face aos persistentes fenômenos de seca e sua associação. Diante disto, o objetivo do presente estudo é analisar o comportamento da precipitação pluviométrica na Mesorregião Sul Cearense e sua relação com o padrão térmico dos oceanos Atlântico e Pacífico. Foram levantados dados pluviométricos de 21 estações. Posteriormente, foram gerados índices de precipitação pluviométrica, entre eles o DCS, DCU, PCPRTOT. Os índices foram correlacionados com as anomalias de TSM do Atlântico e Pacífico. Os resultados indicam que a porção leste da Mesorregião Sul Cearense apresenta aumento no número de dias consecutivos secos. Quanto a precipitação total anual, verificou-se redução dos volumes pluviométricos. Verificou-se que os índices DCS, DCU e PCPRTOT foram bastante influenciados pelas anomalias de TSM do Atlântico e Pacífico. No que se refere aos dois primeiros, a maior influência se deu na porção central e leste da região. Quanto ao PCPRTOT, 99\% dos postos correlacionaram-se com as variações de TSM. Deste modo, constatou-se que a participação dos oceanos Atlântico e Pacífico foram muito importantes para as configurações do regime pluvial verificado na região.
\end{abstract}

Palavras-chave: temperatura de superfície do Mar, eventos de seca, El Niño.

\section{Variability Space-Time of Precipitation Trends in South Mesoregion Cearense and its Relationship with the Faults TSM}

\begin{abstract}
Concern about climate change has raised a number of issues related to precipitation extremes in the Brazilian semiarid region increasingly discussed face persistent drought phenomena and their association. Given this, the objective of this study is to analyze the behavior of rainfall in Southern Mesoregion Cearense and its relation to the thermal pattern of the Atlantic and Pacific oceans. Later, they were generated rainfall rates, including DCS, DCU, PCPRTOT. The indices were related to SST anomalies in the Atlantic and Pacific. The results indicate that the eastern portion of Mesoregion South Cearense presents increase in dry consecutive days. The total annual rainfall, there was a reduction in rainfall volumes. It was found that the DCS indices, DCU and PCPRINT were heavily influenced by the SST anomalies in the Atlantic and Pacific. With regard to the first two, the greatest influence is given in the central portion and the east region. As to PCPRINT $99 \%$ of the posts relate to SST variations. Thus, it was found that the participation of the Atlantic and Pacific oceans were very important to the settings of rainfall patterns found in the region.
\end{abstract}

Keywords: sea surface temperature, drought events, El Niño.

Autor de correspondência: Pedro dos Santos Ferreira, pedro_spe@hotmail.com. 


\section{Introdução}

Nas últimas décadas, o tema mudanças climáticas é um dos mais debatidos pela comunidade científica, gestores públicos e sociedade em geral. A preocupação com a questão encontra-se atrelada aos possíveis impactos que o referido fenômeno pode causar sobre os sistemas naturais e socioeconômicos. De acordo com os dados publicados pelo Intergovernmental Panel on Climate Change (IPCC), espera-se que o aquecimento global, entre outros efeitos, modifique a distribuição espacial e temporal da água, intensificando os problemas de escassez hídrica em regiões onde a oferta é superada pela demanda (IPCC, 2013).

Dentro deste contexto, vários pesquisadores vêm monitorando o comportamento da precipitação em todo o mundo. Os resultados encontrados revelam condições diversas. Para o Oriente Médio e Ásia (Tabari e Talaee, 2011; Fallah et al., 2016), maior parte do continente africano (Bates et al., 2008; Tettey et al., 2017), parte dos Estados Unidos e continente europeu (Gallant et al., 2014; Luce, 2018; Venetsanou et al., 2016), observa-se, de modo geral, tendência de redução dos valores de precipitação pluvial. Quanto a América do Sul, o padrão detectado por Skansi et al. (2013) indica aumento da condição de umidade para a porção sudeste da região.

No Brasil, o Nordeste é uma das regiões mais problemáticas no que se refere à disponibilidade de água. Isto porque a região conta com uma grande área semiárida, que representa $53 \%$ do território nordestino, onde as chuvas são bastante escassas e irregulares. Nos últimos anos, os recorrentes e persistentes fenômenos de seca que assolaram a região levantaram questões relacionadas aos possíveis efeitos das mudanças climáticas. Todavia, a ideia perde força em função das próprias características naturais da região e seu histórico de variabilidades climáticas atreladas à sazonalidade dos sistemas atmosféricos atuantes na região.

De acordo com Assis et al. (2015), o regime de chuvas no semiárido é controlado pela atuação de sistemas atmosféricos como instabilidades associadas às Frentes Frias, Vórtices Ciclônicos de Ar Superior, (VCAS) e Zona de Convergência Intertropical (ZCIT). Entre os mencionados, a ZCIT é um dos mais influentes sobre a região, sendo decisivo para as configurações de anos secos ou muito secos, devido ao dipolo positivo do Atlântico que favorece o descolamento da ZCIT para posições mais ao norte da linha do Equador. Os efeitos são agravados em anos coincidentes com os eventos de El Niño (Marengo, 2007; Marengo et al., 2016; Nóbrega e Santiago, 2014).

Os sistemas atmosféricos mencionados resultam da variabilidade de Temperatura de Superfície do Mar (TSM) do Atlântico e Pacífico. Em relação ao Atlântico, o padrão de anomalias de TSM detectados por Hastenrath e Heller (1977) demonstram que nos anos em que a região Equatorial e Tropical do Oceano é mais baixa em comparação ao Norte (Fase Positiva do Dipolo), verifica-se inibição da formação de nuvens sobre o Nordeste brasileiro, contribuindo para a diminuição da precipitação pluvial. Os efeitos dessa dinâmica (secas sobre o Nordeste) são intensificados quando se observa padrão anômalo de circulação atmosférica no Pacífico. Neste caso, percebeu-se um aquecimento das águas superficiais acima da média histórica da porção tropical do referido Oceano, mecanismo que altera o posicionamento da célula de Walker, inibindo a formação de nuvens sobre o Nordeste. Este fenômeno é denominado El Niño.

Em resposta a este evento atmosférico, verifica-se uma variação da pressão atmosférica tropical, denominada Oscilação Sul (OS). A OS é geralmente analisada por meio de um índice, neste caso Southern Oscillation Index (SOI). De acordo com Nóbrega e Santiago (2014) o SOI representa a diferença entre os desvios normalizados da pressão na superfície entre as porções localizadas no Tahiti (Pacífico Central-sul) e Darwin (norte da Austrália).

Numerosos estudos desenvolvidos nos últimos anos têm comprovado a influência das anomalias de TSM do Atlântico e Pacífico tropicais sobre a precipitação pluviométrica no Nordeste brasileiro. Um deles é o desenvolvido por Araújo e Brito (2011), para os estados da Bahia e Sergipe. Os autores utilizaram dados diários de precipitação referentes a um período de 45 anos e em 75 estações pluviais, para os quais os mesmos geraram e analisaram índices como número de Dias Consecutivos Secos (DCS), número de Dias Consecutivos Chuvosos (DCU) e Precipitação Total (PRCPTOT). Os índices analisados apresentaram correlação com ao menos um dos setores dos Oceanos Atlântico e Pacífico, isto com um intervalo de significância entre 90 e $95 \%$.

Para o estado de Pernambuco, Nóbrega e Santiago (2014) analisaram o padrão de TSM dos Oceanos Atlântico e Pacífico, entre 1950 e 2007, onde relacionaram com as variabilidades pluviométricas da precipitação pluvial. Os resultados obtidos pelos autores demonstraram que houve, para o período analisado, uma tendência positiva de aquecimento para ambos os oceanos. Este comportamento produziu impactos sobre a variabilidade climática na região.

Outro estudo que merece destaque foi o conduzido por Nóbrega et al. (2016) para todo o Nordeste. Os autores investigaram os padrões pluviométricos da região e correlacionaram com as anomalias de TSM do Atlântico e Pacífico. Para avaliação da tendência de temperatura dos oceanos mencionados empregaram o teste de Mann-Kendal. Onde utilizaram o Índice de Anomalia de Chuva (IAC) para evidenciar as relações. Os resultados demonstraram que nos últimos anos vêm ocorrendo mudanças nos padrões de precipitação pluviométrica. Porém, os autores expõem que este padrão tem relação com as anomalias positivas do Pacífico Leste (El Niño) e negativas sobre o Atlântico Sul (Dipolo do Atlântico). Este quadro, segundo os autores, contribui para a configuração de anos onde se verificam secas mais severas. 
A variabilidade espacial da precipitação no semiárido nordestino torna sua análise um grande desafio, principalmente quando se insere nas discussões temas tão complexos como o das mudanças climáticas. Por essa razão e diante dos fatos aqui discutidos, o objetivo principal do presente estudo é analisar o comportamento da precipitação pluviométrica na Mesorregião Sul Cearense e sua relação com o padrão térmico dos oceanos Atlântico e Pacífico.

\section{Material e Métodos}

\section{1. Área de estudo}

A área em estudo é reconhecida devido ao seu rico patrimônio físico e ambiental, considerado como ambiente estratégico para o estado do Ceará. Situada na porção sul, faz divisa com as Mesorregiões Centro Sul e Sertões no estado do Ceará e os estados de Pernambuco, da Paraíba e Piauí (Fig. 1).

Em sua totalidade é formada por 25 municípios (Abaiara, Altaneira, Araripe, Assaré, Aurora, Barbalha, Barro, Brejo Santo, Campos Sales, Caririaçu, Crato, Farias Brito, Granjeiro, Jardim, Jati, Juazeiro do Norte, Mauriti, Milagres, Missão Velha, Nova Olinda, Penaforte, Porteiras, Potengi, Salitre e Santana do Cariri), apresentan- do 876.600 habitantes, aproximadamente $10,4 \%$ da população do estado (IBGE, 2010).

\subsection{Procedimentos metodológicos}

\subsubsection{Aquisição e tratamento dos dados}

Os dados de precipitação pluviométrica utilizados na presente pesquisa foram obtidos junto a Agência Nacional de Água (ANA). Ao todo, selecionou-se 21 postos pluviométricos com informações, para os quais a precipitação medida encontra-se em escala temporal diária e o período estende-se de 1963 a 2014, totalizando 51 anos. O critério adotado para escolha destes postos foi o de percentual de falhas, onde o limiar adotado foi de até $5 \%$. Como o clima dominante na área de estudo é o tropical semiárido, optouse por não realizar preenchimento das falhas observadas, uma vez que, dada a variabilidade temporal e espacial da precipitação, observou-se erros significativos para correção de dados faltosos para o período chuvoso. Por outro lado, o percentual de falhas é relativamente baixo, o que não implica na confiabilidade dos resultados. Quanto a qualidade dos dados, realizou-se tratamento simples, onde procurou-se identificar e eliminar valores de precipitação não

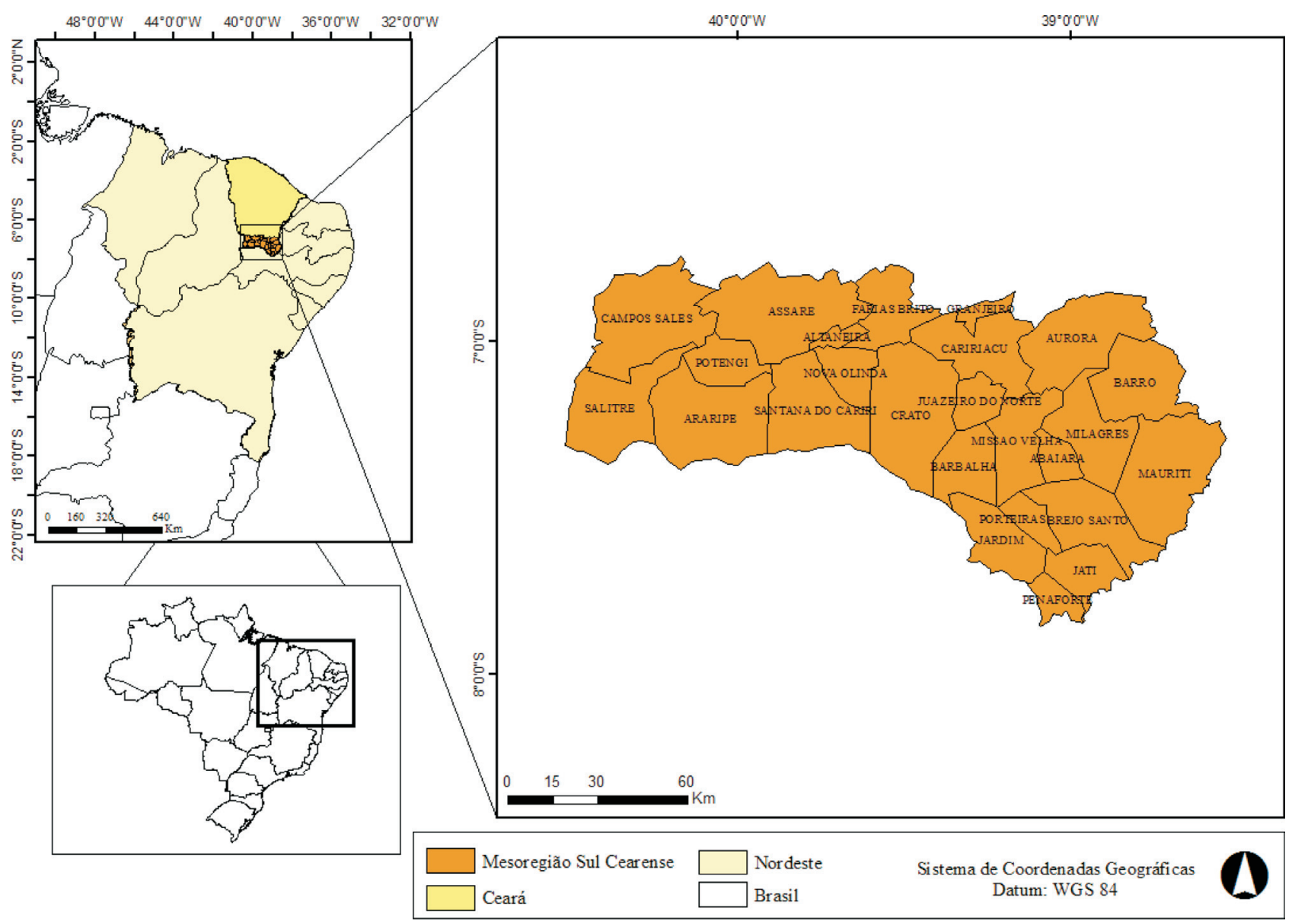

Figura 1 - Localização geográfica da Mesorregião Sul Cearense. 
condizentes com a realidade do local, aqueles provavelmente relacionados a erros na coleta ou transmissão.

\subsection{2. Índices dependentes da precipitação}

Na Tabela 1 encontra-se a descrição dos índices climáticos derivados da precipitação pluviométrica utilizados na presente pesquisa. Os mesmos são recomendados pela Expert Team on Climate Change Detection, Monitoring and Índices (ETCCDMI) para análises de séries históricas de precipitação pluvial.

Através do Rclimdex (R), versão 3.2.1 calculou-se os índices DCS, DCU e PRCPTOT. O software foi desenvolvido por Byron Gleason do National Climate Data Centre (NCDC) e é utilizado para gerar índices relacionados aos extremos climáticos, os quais podem ser utilizados em estudos que buscam monitorar ou detectar possíveis mudanças de ordem climática (Souza e Azevedo, 2012). A saída do R fornece um conjunto de dados estatísticos referentes à tendência linear calculada pelo método de mínimos quadrados, coeficiente de determinação $\left(\mathrm{R}^{2}\right)$, erro padrão de estimativa e tendência linear, assim como o nível de significância estatística dos mesmos. Para definição dos dias classificados como úmidos ou secos, adotou-se o limiar $1 \mathrm{~mm}$. Ou seja, em dias com chuvas inferiores a $1 \mathrm{~mm}$, estes são classificados como secos. Para os dias úmidos, a precipitação é superior a $1 \mathrm{~mm}$.

\subsubsection{Teste de Mann-Kendall}

O teste Mann-Kendall (MK), proposto inicialmente por Sneyers (1975), foi aplicado neste estudo para detectar possíveis alterações de ordem climática nas séries temporais de precipitação da mesorregião Sul Cearense. De acordo com Goossens e Berger (1986) este teste é o mais recomendado para estudos dessa natureza, visto que permite identificar, aproximadamente, onde o ponto da mudança se inicia.

Por meio do método sequencial de $\mathrm{MK}$, são testadas as hipóteses $\left(H_{0}\right.$ e $\left.H_{1}\right)$ para tendência de séries temporais de longo prazo. Assim, a hipótese é nula $\left(H_{0}\right)$ quando não se observa tendência de mudança na população de um determinado conjunto de dados $\left(X_{1}, X_{2}, \ldots, X_{n}\right)$. Já a hipótese $H_{1}$ refere-se à detecção de alguma tendência monotônica observada no tempo, sendo assim, rejeita-se a hipótese $H_{0}$.

Considerada uma determinada série temporal, $x_{i}$ de $N$ termos $(1 \leq i \leq n)$, o MK é determinado por meio da soma $t_{n}$ de um conjunto de termos $m_{i}$, relativo ao valor $x_{i}$, pelo qual

Tabela 1 - Definições e unidades dos índices gerados a partir da precipitação pluviométrica.

\begin{tabular}{lll}
\hline Índice & Descrição & Unidade \\
\hline DCS & Dias Consecutivos Secos & dias/ano \\
DCU & Dias Consecutivos Úmidos & dias/ano \\
PRCPTOT & Precipitação Total Anual & $\mathrm{mm} / \mathrm{ano}$ \\
\hline
\end{tabular}

os termos anteriores $(j<i)$ são inferiores ao mesmo, conforme descrito na Eq. (1):

$$
\text { tn }=\sum_{i=1}^{n-1} \sum_{j-i+1}^{n} \operatorname{sgn}\left(x_{j}-x_{i}\right)
$$

Em séries temporais com grande número de termos $(N)$, sob a hipótese nula $H_{0}$ de ausência de tendência, $t_{n}$ apresentará uma distribuição normal com média $E(t n)=0$ e variância (Eq. (2)):

$$
V A R_{t n}=\sqrt{(n / 18)(n-1)(2 n-5)}
$$

Para rejeição da hipótese nula, é aplicado o teste de significância estatística de $t_{n}$ por meio de um teste bilateral para grandes valores $U\left(t_{n}\right)$, por meio de (Eq. (3)):

$$
U(t n)=(t n-E(t n)) /(\operatorname{var}(t n)) 1 / 2
$$

Na versão sequencial, duas curvas $\left(U(t n)\right.$ e $\left.U^{*}(t n)\right)$ e o seu inverso, são geradas. Deste modo, como resultado da interação entre as mesmas, obtêm-se o ponto aproximado da mudança abrupta na mediana da série, caso o valor encontre-se dentro do intervalo de confiança estatística $(\alpha=0,05)$. Ou seja, o ponto aproximado do início de uma tendência é detectado quando a curva U(tn) cruza os limites de confiança ao nível de $95 \%$.

Através do Climap executou-se os cálculos do teste sequencial de MK. O programa foi desenvolvido por Salvador (2014) para a análise da variabilidade climática na nova fronteira agrícola do Brasil: região do Matopiba. O Software apresenta interface gráfica simples e amigável, onde o mesmo é elaborado a partir de linguagem Python. O Climap pode ser adquirido gratuitamente junto a UFCG.

A etapa final consistiu na geração dos mapas para espacialização dos índices de precipitação e teste sequencial de MK. Para este fim, utilizou-se o software de geoprocessamento ArcGis 9.3, licenciado junto ao laboratório de Sensoriamento Remoto e Geoprocessamento (SERGEO), locado na Universidade Federal de Pernambuco (UFPE).

\subsection{4. Índices de precipitação e anomalias de TSM}

Os índices oceânicos utilizados correspondem aos medidos no Pacífico e no Atlântico nas regiões de Niño 1+2 $\left(0-10^{\circ} \mathrm{S}, 90^{\circ} \mathrm{W}-80^{\circ} \mathrm{W}\right)$, Niño $3\left(5^{\circ} \mathrm{N}-5^{\circ} \mathrm{S}, 150^{\circ} \mathrm{W}-90^{\circ}\right.$ $\mathrm{W})$, Niño $3.4\left(5^{\circ} \mathrm{N}-5^{\circ} \mathrm{S}, 170^{\circ} \mathrm{W}-120^{\circ} \mathrm{W}\right)$ e Niño 4 $\left(5^{\circ} \mathrm{N}-5^{\circ} \mathrm{S}, 160^{\circ} \mathrm{E}-150^{\circ} \mathrm{W}\right)$, como também o Índice de Oscilação Sul (IOS) - entre as ilhas australianas de Tahiti e Darwin no oceano Pacífico equatorial e o Índice Oceânico Niño (ION) - na região Niño 3.4 no oceano Pacífico equatorial. Na área do oceano Atlântico utilizou-se o Índice mensal do Atlântico Tropical Norte (TNAI), que é a anomalia de TSM na área compreendida entre $5^{\circ} \mathrm{N}-23,5^{\circ} \mathrm{N}$ e $15^{\circ} \mathrm{W}-57,5^{\circ} \mathrm{W}$, e o Índice mensal do Atlântico Tropical Sul (TSAI), que é a anomalia de TSM na área compreendida entre $0-20^{\circ} \mathrm{S}$ e $10^{\circ} \mathrm{E}-30^{\circ} \mathrm{W}$. Os índices oceânicos 
medidos no Pacífico e no Atlântico foram fornecidos por National Oceanic and Atmospheric Administration (NOAA), no período de 1963 a 2014.

Os índices utilizados para análise de influência das anomalias de TSM sobre a precipitação para o período analisado (1963-2014) foram DCS, DCC e PRCPTOT, onde os mesmos foram correlacionados com os dados de temperatura de superfície do mar dos setores Niño $1+2$, Niño 4, Niño 3,4 e Niño 3, do Pacífico tropical, como também os índices IOS e ION. Para a região do Atlântico tropical, os dados correspondentes aos índices de precipitação foram correlacionados com os índices TNA e TSA. Para isto, aplicou-se a correlação de Pearson, onde também se levou em consideração o nível de significância da mesma dentro de um intervalo de confiança de 90 e $95 \%$.

\section{Resultados e Discussão}

\section{1. Índices de climáticos dependentes da precipitação}

O resumo dos resultados obtidos para os índices número de dias consecutivos secos (DCS) e número de Dias Consecutivos Úmidos (DCU) pode ser observado nas Figs. 2 e 3. Ressalta-se que para esta avaliação considerou-se como tendência estatisticamente significante os intervalos cujo p-valor é igual ou inferior a 0,05 , ou seja, dentro de um intervalo de confiança ao nível de $95 \%$. Dos 21 postos pluviométricos utilizados no presente estudo, cinco $(23,8 \%)$ apresentaram tendência de aumento para o DCS, concentrados principalmente na porção leste da região. As estações Brito Farias e Aurora são as únicas com tendência negativa dentro do intervalo de significância estatística. Os resultados desses índices, comparados aos obtidos a partir da estimativa do número de dias consecutivos úmidos (DCU), observado na Fig. 3, revelam que para três estações pluviais (Altaneira, Aurora e Jardim), comportamento contrário em relação ao sinal de tendência dos índices. Para Altaneira e Jardim, por exemplo, constata-se que as localidades vêm se tornando mais áridas. Enquanto para Aurora, observa-se aumento da condição de umidade.

Os resultados observados para os dois índices, de modo geral, divergem dos encontrados por Haylock et al. (2006) em estudo realizado para a América do Sul. Os autores utilizaram três estações para a região Nordeste do Brasil, as quais demonstraram aumento da condição de umidade sobre a região.

Vale ressaltar que a divergência dos resultados encontrados na presente pesquisa em relação aos estudos citados, em parte, pode estar associada à variabilidade espacial da precipitação no Semiárido. Como nos estudos realizados por Haylock et al. (2006) o número de postos pluviométricos é proporcionalmente à área, os fenômenos atrelados a

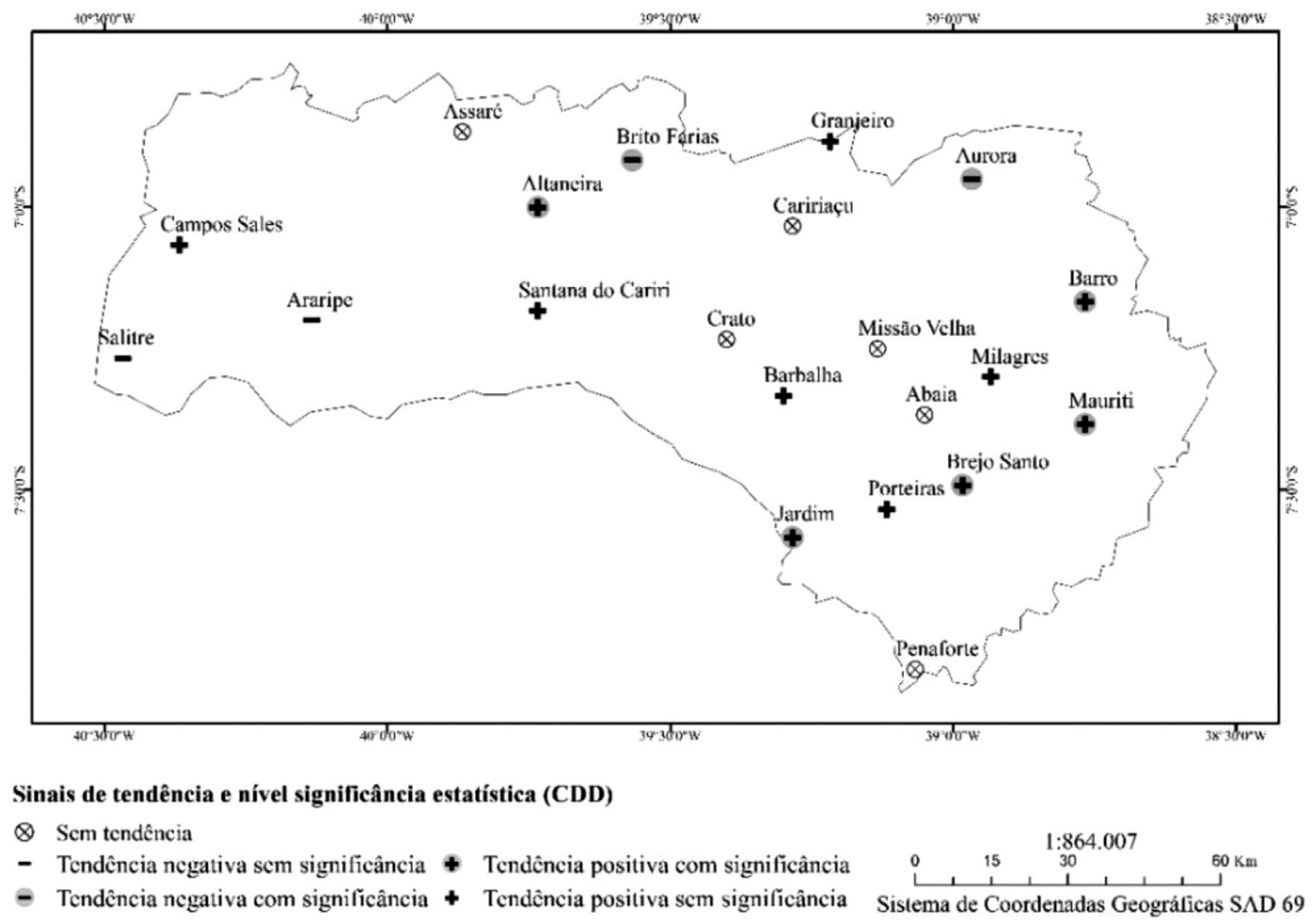

Figura 2 - Distribuição espacial dos sinais e nível de significância estatística para o índice número de dias consecutivos secos (DCS) na mesorregião Sul Cearense. 


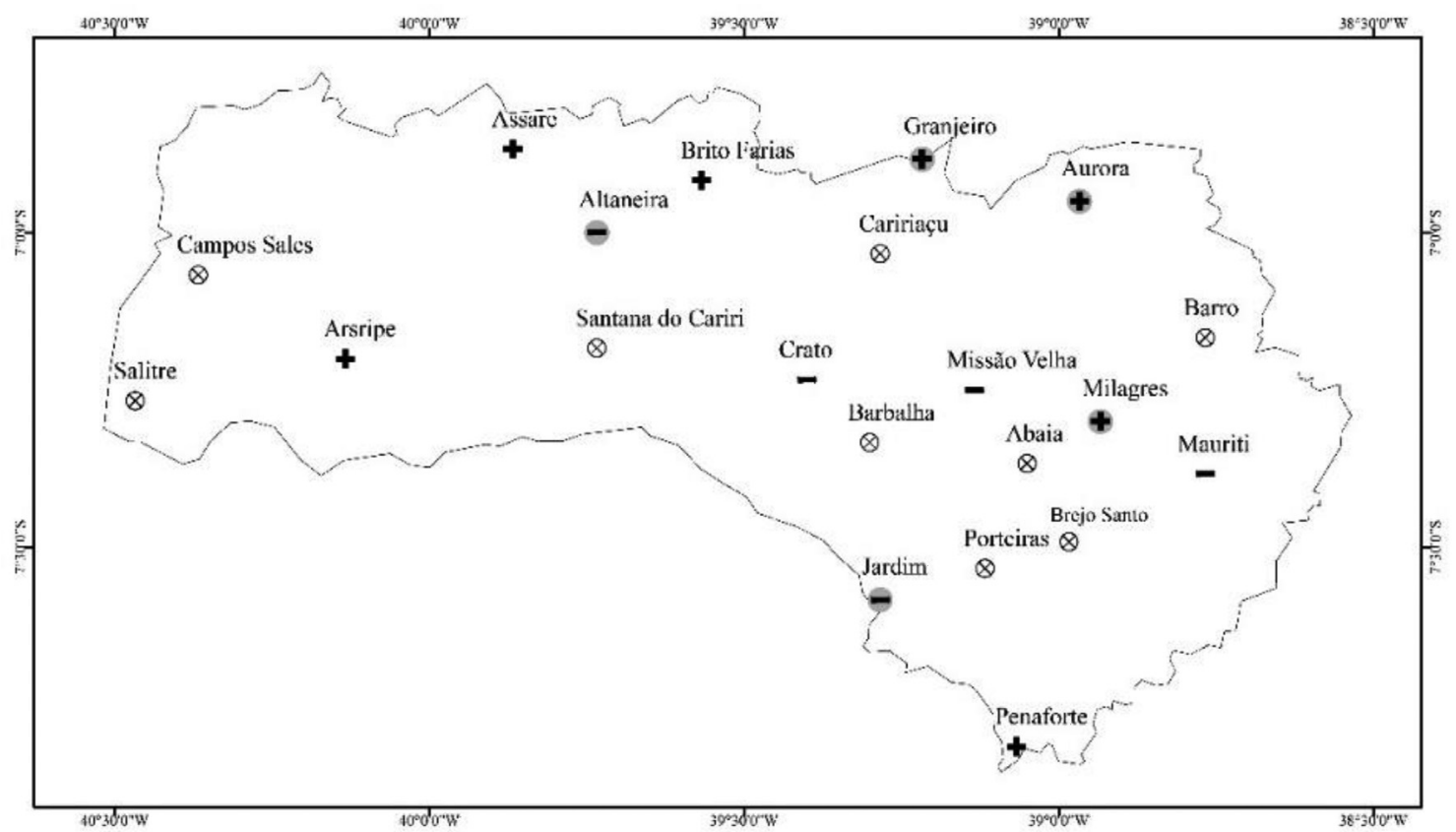

Sinais de tendência e nível significância cstatistica (CWD)

Q Sem tendência

- Tendência negativa sem significância — Tendência positiva conı significância

- Tendência negativa com significância + Tendência positiva sem significância

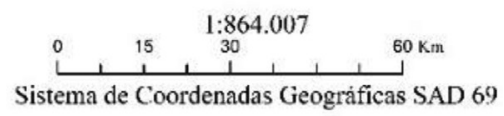

Figura 3 - Distribuição espacial dos sinais e nível de significância estatística para o índice número de dias consecutivos secos (DCU) na mesorregião Sul Cearense.

variabilidade espacial das chuvas são minimizados. Quando os resultados encontrados na presente pesquisa são comparados aos expostos por Santos et al. (2013), que utilizaram maior quantidade de postos pluviométricos (26) para o estado de Pernambuco, os resultados são convergentes.

Deste modo, quando considerado tal fator, para outros estudos onde se observa maior detalhamento espacial, à exemplo do realizado por Santos et al. (2013), verifica-se convergência nos resultados encontrados.

Quanto ao índice PRCPTOT, este é exposto na Fig. 4. Sem que se leve em consideração o nível de significância estatística, observa-se que $70 \%$ dos postos pluviais apresentam tendência de redução dos totais pluviométricos anuais para suas séries históricas. Para as demais estações, a maior parte delas $(33,3 \%)$ não apresentou tendência, enquanto a estação de Bento Farias é a única que indica tendência positiva, porém, sem significância. Quando considerados os postos cujo p-valor encontra-se dentro do intervalo de confiança ao nível de $95 \%$, o percentual de estações com tendência negativa cai de $70 \%$ para $28,5 \%$, concentradas principalmente na porção leste. No geral, esses resultados são contrários aos encontrados por Santos et al. (2009), que identificaram tendência de incremento na precipitação pluvial no estado do Ceará, assim como com os encontrados por Haylock et al. (2006).

\subsection{Mann-Kendall}

A espacialização das tendências para o teste sequencial de MK, empregado para análise da precipitação total anual, pode ser visualizada na Fig. 5. Observa-se que o padrão dos sinais é semelhante ao verificado com auxílio do índice PRCPTOT, porém com redução no quantitativo de estações com tendência negativa quando considerado o valor $\mathrm{p} \leq 0,05$. Diagnosticou-se que cerca de $57 \%$ dos postos pluviais possuem tendência negativa em suas séries históricas, porém, esse percentual é reduzido a 19\% quando considerado o intervalo de significância estatística ao nível de $95 \%$. As estações localizadas em Brejo Santo e Muriti não apresentaram significância estatística para o teste de MK como observado para o PCRPTOT. Vale ressaltar que o teste sequencial de MK é considerado mais indicado para detecção de possíveis mudanças relacionadas ao padrão de precipitação pluvial. De acordo com Goossens e Berger (1986), o teste de MK é considerado mais criterioso ao nível de análise, sendo o mais recomendado para estudos climatológicos devido a sua capacidade de detecção e localização aproximada do ponto inicial em que uma determinada tendência se inicia.

Na Fig. 6 são exibidas as séries temporais de precipitação pluviométrica para o teste sequencial de MannKendall. Todavia, só foram representados os postos que apresentaram tendência com significância estatística para 
$40^{\circ} 390 \mathrm{c} w$ $40^{\circ} 00^{\circ} \mathrm{W}$ $39^{\circ} 39^{\circ 0^{\circ} \mathrm{W}}$ $30000 \mathrm{~W}$ $38^{\circ} 30^{\circ} 0^{4} \mathrm{~W}$

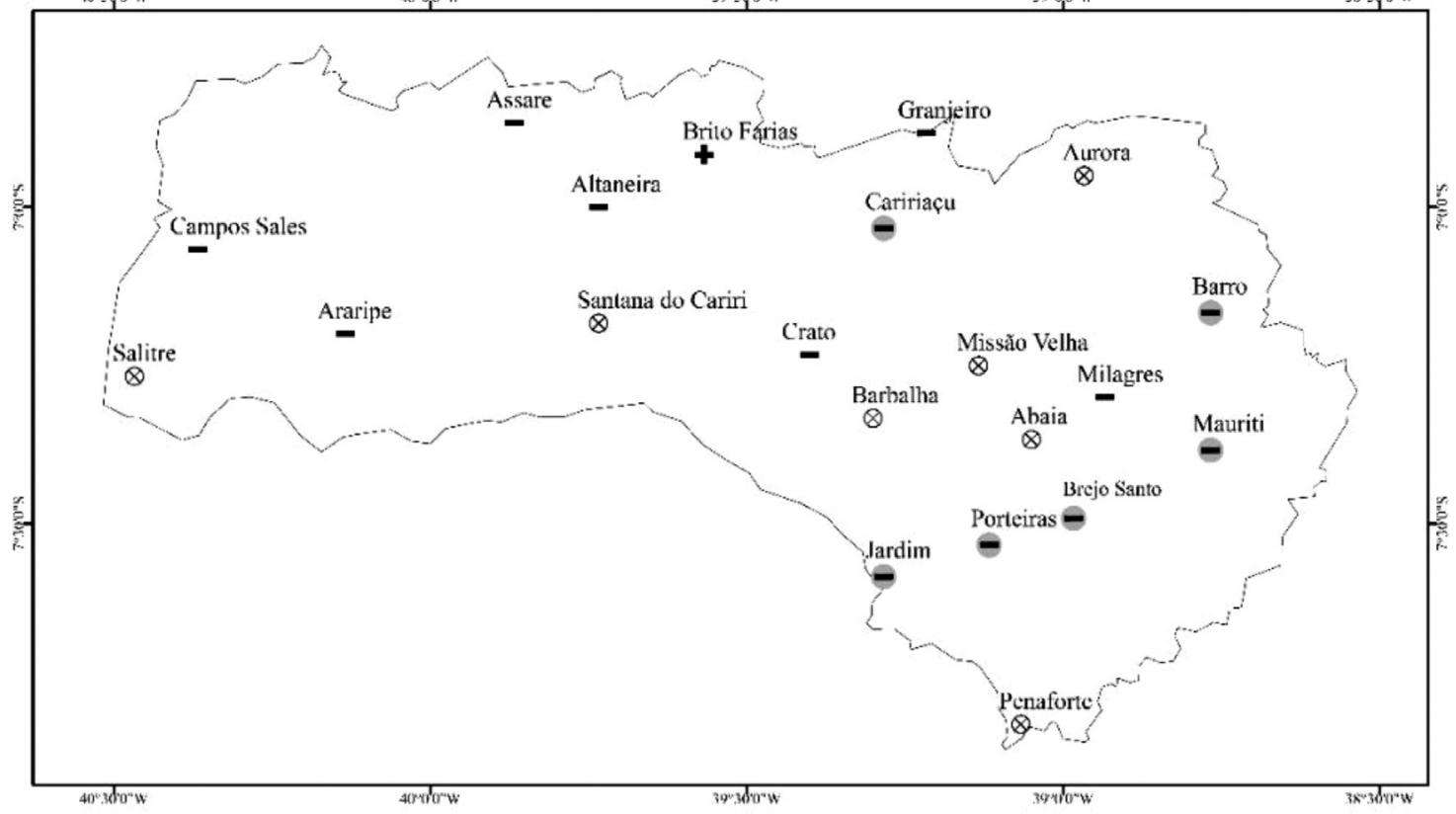

\section{Sinais de tendência c nível significância estatistica (PRCPTOT)}

$\otimes$ Sem tendência

- Tendência negativa scm significância † Tendência positiva com significância

- Tendência negativa com significância + Tendência positiva sem significância

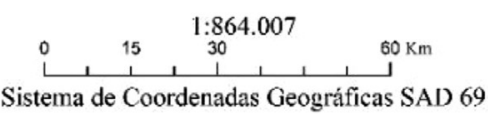

Figura 4 - Distribuição espacial dos sinais e nível de significância estatística para o índice precipitação total mm/ano (PRCPTOT) na mesorregião Sul Cearense.

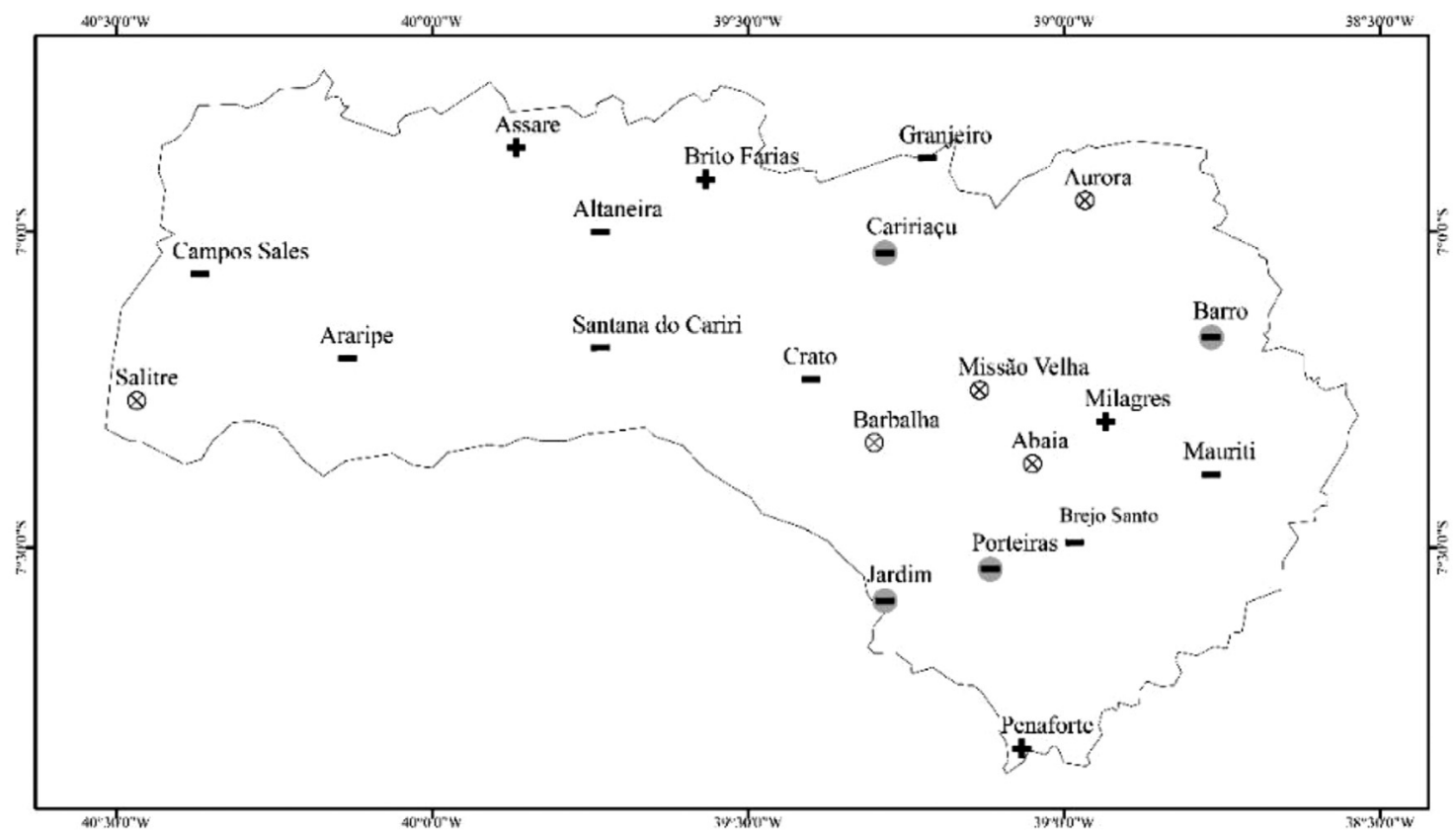

Sinais de tendência c nível signifīcância estatistica (Mann Kendall)

$\otimes$ Sem tendência

- Tendência negativa sem significância † Tendência positiva com significância

- Tendência negativa com significância + Tendência positiva sem significância

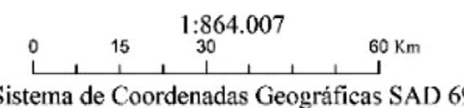

Figura 5 - Distribuição espacial dos sinais e nível de significância estatística para o teste sequencial de Mann-Kendall na mesorregião Sul Cearense. 
suas séries temporais. Dentre as estações, apenas três possibilitam a realização dessa análise (Barro, Caririaçu e Jardim), já que para o posto de Porteiras, apesar de apresentar significância estatística, a linha $U t$ não pôde ser gerada devido quantidade de falhas, o que impossibilita determinar quando se inicia a mudança.

De modo geral, os três postos para os quais a linha $U t$ é gerada, indicam que a mudança no padrão de precipitação total tem início no final de década de 1980, com exceção para Caririaçu, que se inicia já em 1970. Observa-se que após a linha $U t$ cruzar o limite de significância estatística, a mesma se mantem abaixo do referido limiar até os anos mais recentes, caracterizando uma mudança no padrão das chuvas nessas localidades. Por outro lado, é importante ressaltar que o período referente aos meados dos anos de 1970 e final da década de 1980, é considerado bem atípico devido a recorrência de anos muito secos.

De acordo com Campos (2004), de 1973 a 1983 o Nordeste brasileiro foi atingido por longos períodos de escassez de chuvas, continuando de maneira aleatória entre os anos de 1997, 1998, 2003, 2004 e 2005. Deste modo, ressalta-se que o padrão observado não reflete necessariamente uma mudança de ordem climática. Ademais, Marengo (2007) destaca que os períodos muito secos ou muito chuvosos no semiárido nordestino alternam-se aleatoriamente, sob forte influência da atuação dos sistemas atmosféricos resultantes do padrão termodinâmico dos oceanos Pacifico e Atlântico tropicais.

O aumento do número de dias consecutivos secos e a redução dos totais pluviométricos observados em algumas localidades representam graves implicações para os recur-
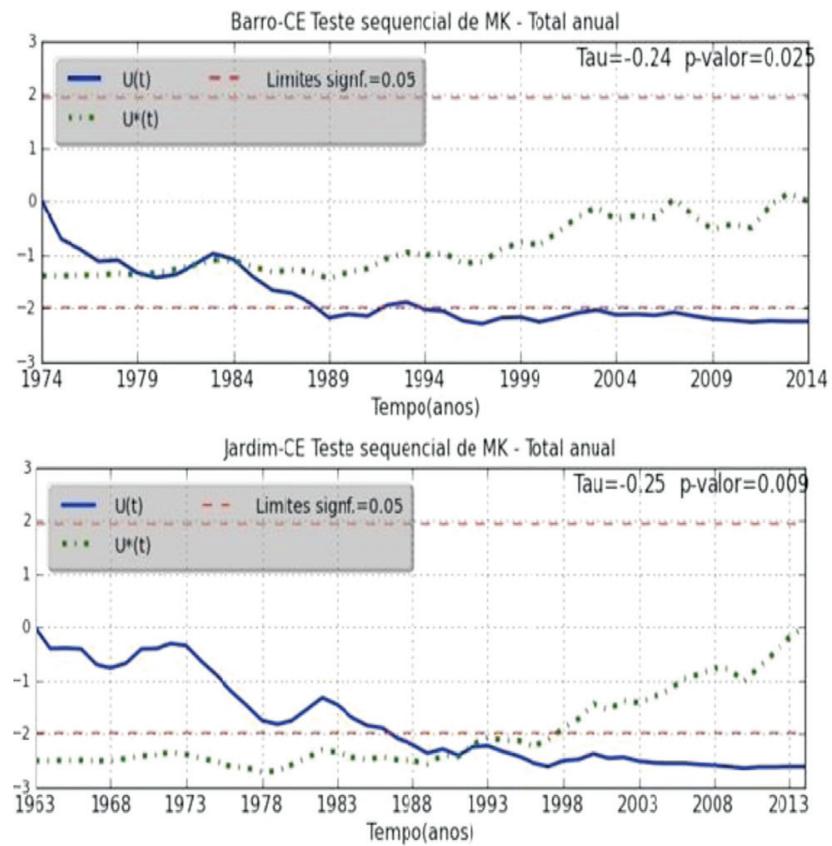

sos hídricos e os sistemas socioeconômicos. Os principais impactos recaem sobre a redução da disponibilidade de água tanto para o abastecimento humano quanto para o desenvolvimento de práricas agricolas. No Semiárido, este dinamismo, atrelado a gestão inadequada dos recursos hídricos, é apontado como principal responsável para os problemas relacionados a perdas de vida humana e prejuízos materiais, problemas que assolam a região há décadas.

\subsection{Correlação dos índices dependentes da precipitação pluvial com as anomalias de TSM}

Dada a influência dos oceanos Pacífico e Atlântico tropicais sobre o padrão das chuvas no semiárido nordestino (Nóbrega e Santiago, 2014), optou-se por correlacionar os índices DCS, DCU e PCRPTOT com as anomalias de TSM para os setores do Atlântico e Pacífico. Os resultados obtidos para o índice DCS podem ser visualizados na Tabela 2, a qual expõe o valor das correlações, assim como seus níveis de significância estatística. Quando considerado apenas o limiar de $95 \%$ de confiança, verificou-se que $48 \%$ dos postos pluviométricos apresentaram correlação estatisticamente significante. Já para os postos cujo nível de significância da correlação foi de $90 \%$, desconsiderando os que ultrapassaram este limite, verifica-se que estes correspondem a $38 \%$ do total. Ou seja, $86 \%$ dos postos pluviométricos apresentaram tendência com significância entre 90 e $95 \%$.

Entre os postos pluviais que indicaram tendência para o DCS, seja positiva ou negativa, apenas o Mauriti não possui correlação estatisticamente significante $(90 \%$ ou $95 \%)$ para um dos índices ou setores relativos aos oceanos Atlân-
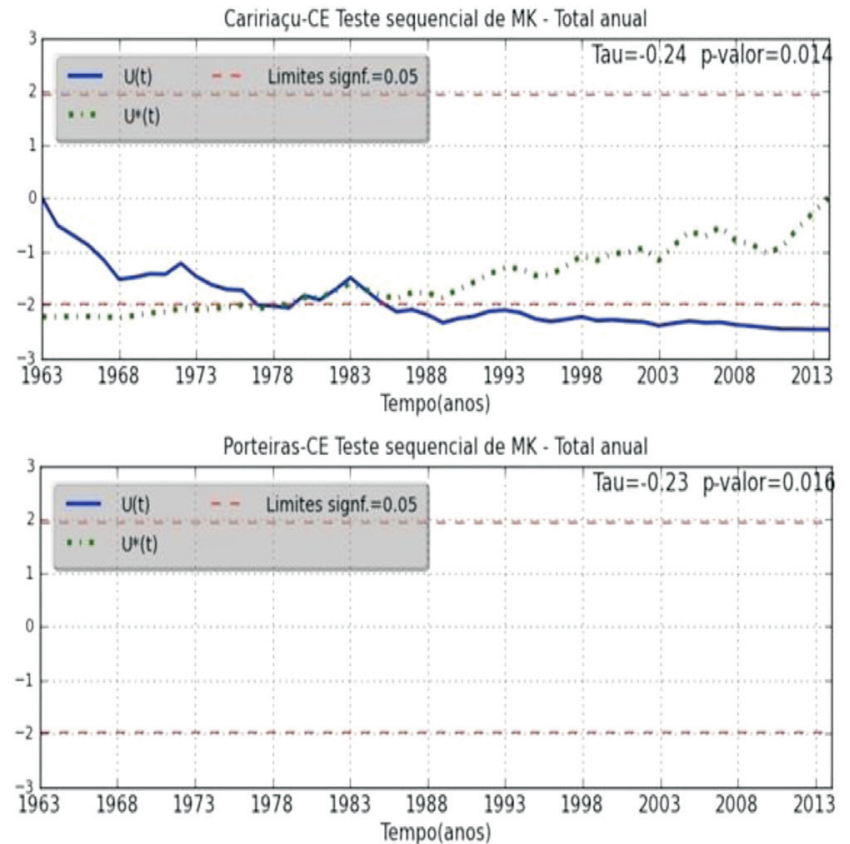

Figura 6 - Gráficos do teste sequencial de MK (estações que apresentaram significâncias estatística) para precipitação pluviométrica total anual dos postos de Barros-CE, Caririaçu-CE, Jardim-CE e Porteiras-CE. 
tico (TNA e TSA) e Pacífico (SOI, ONI, Niño 1 +2, Niño 3, Niño 3.4 e Niño 4). Quanto aos oceanos que mais influenciaram o comportamento da precipitação para este período, para os postos de Brito Farias e Brejo Santo houve maior correlação estatisticamente significantes com setores do Pacífico. No que se refere às estações Altaneira, Aurora, Barro e Jardim, suas séries pluviais foram bastante influenciadas pelas anomalias de TSM do Atlântico. Salienta-se que o posto de Aurora foi o único que apresentou tendência negativa para o DCS, indicando que, de maneira geral, o crescente número de dias consecutivos secos observados nas outras estações está relacionado à sazonalidade da ZCIT.

Quanto ao índice DCU, 52\% dos postos pluviométricos correlacionaram-se com as variações de TSM do Atlântico e Pacífico, isto considerando o intervalo de confiança a 90 e 95\%, conforme observado na Tabela 3. Ao nível de $95 \%$ de confiança, o percentual de estações correlacionadas cai para $38 \%$. Entretanto, a série do posto pluviométrico localizado em Altaneira é uma das que foram mais influenciadas por todos os setores e índices de oscilação do Atlântico e Pacífico, mesmo quando se leva em consideração apenas os intervalos de significância ao nível de $95 \%$. Isto ratifica a importância dos sistemas atmosféricos resultantes das anomalias de TSM dos referidos oceanos para as preci- pitações na região. Outro ponto evidente é a variabilidade espacial da influência.

Na Tabela 4 é exposto o resumo dos resultados referentes à correlação do índice PRCPTOT com os dados de TSM. Como pode ser observado, em relação ao DCS e DCU, este índice apresentou maior quantidade de estações cujas séries temporais correlacionam-se com as anomalias de TSM. Todas as séries temporais geradas pelas estações pluviais possuem valores de correlação acima de $20 \%$, com significância estatística variando entre 90 e $95 \%$, para pelo menos um dos setores dos oceanos Atlântico e Pacífico. Quando se leva em consideração apenas os postos cuja a correlação encontra-se dentro de um intervalo de confiança de $95 \%$, apenas o de Araripe não se enquadra dentro deste limiar. Outro ponto que diferencia o posto de Araripe dos demais diz respeito à influência dos setores e índices relativos às anomalias de TSM, uma vez que este é o único que não se correlaciona ( $90 \%$ ou $95 \%$ de confiança), simultaneamente, com os valores de TSM dos oceanos.

De modo geral, é possível observar que os maiores valores de correlação e nível de significância estatística foram observados para o índice PRCPTOT. Isto se deve ao fato de que o DCC e DCU são calculados em escala diária, ou seja, sofrem controle de outros sistemas de menor escala que atuam na região, a exemplos das instabilidades associa-

Tabela 2 - Correlação do número de dias consecutivos secos (DCS) para a mesorregião Sul Cearense com as anomalias de Temperatura de Superfície do Mar para o período de 1963 a 2014.

\begin{tabular}{|c|c|c|c|c|c|c|c|c|}
\hline Estações & SOI & NINO 3 & TNA & TSA & ONI & Nino1+2 & Nino 4 & Nino 3,4 \\
\hline Altaneira & 0,02 & 0,09 & $0,36^{* *}$ & 0,09 & 0,05 & 0,13 & 0,05 & 0,01 \\
\hline Araripe & 0,17 & 0,1 & 0,14 & $0,23^{*}$ & $0,24^{*}$ & 0,05 & 0,11 & 0,19 \\
\hline Assaré & 0,16 & $0,25^{*}$ & $0,36^{* *}$ & 0,01 & 0,2 & $0,26^{*}$ & 0,21 & $0,22 *$ \\
\hline Aurora & 0,09 & 0,03 & 0,03 & $0,28 * *$ & 0,05 & 0,1 & 0,15 & 0 \\
\hline Barbalha & 0,19 & 0,09 & 0,17 & 0,14 & 0,08 & 0,07 & 0,07 & 0,09 \\
\hline Brejo santo & $0,22 *$ & 0,13 & 0,15 & 0,13 & 0,11 & 0,09 & 0,13 & 0,12 \\
\hline Brito farias & $0,29 * *$ & $0,22 *$ & 0,07 & 0,16 & $0,27 * *$ & 0,18 & 0,13 & $0,22^{*}$ \\
\hline Caririaçu & 0,08 & 0,06 & 0,12 & 0,1 & 0,03 & 0,1 & 0,03 & 0,02 \\
\hline Crato & 0 & 0 & 0,08 & 0,02 & 0,05 & 0,02 & 0,07 & 0,04 \\
\hline Granjeiro & 0,15 & $0,31 * *$ & $0,24^{*}$ & 0,05 & 0,17 & $0,32 * *$ & 0,18 & $0,22 *$ \\
\hline Jardim & 0,05 & 0,19 & $0,29 * *$ & 0,05 & 0,09 & 0,15 & 0,2 & 0,16 \\
\hline Mauriti & 0,05 & 0,1 & 0,17 & 0,11 & 0,01 & 0,11 & 0,04 & 0,04 \\
\hline Milagres & 0,15 & 0,04 & 0,16 & 0,04 & 0,07 & 0,08 & 0,02 & 0,03 \\
\hline Missão velha & 0,05 & 0 & 0,15 & 0,16 & 0,03 & 0,02 & 0,12 & 0,07 \\
\hline Porteira & $0,25^{*}$ & $0,32 * *$ & 0,1 & 0,03 & $0,22 *$ & $0,35^{* *}$ & 0,1 & $0,22 *$ \\
\hline Santana & 0,03 & 0,18 & $0,38 * *$ & 0 & 0,08 & 0,15 & 0,12 & 0,11 \\
\hline Campos Sales & $0,24 *$ & 0,11 & 0,09 & 0,21 & 0,17 & 0,07 & 0,09 & 0,13 \\
\hline Barro & 0,03 & 0,04 & $0,39 * *$ & 0,08 & 0,06 & 0,11 & 0 & 0,03 \\
\hline Abaia & $0,37 * *$ & 0,22 & 0,02 & 0,24 & 0,25 & 0,12 & 0,25 & 0,24 \\
\hline Penaforte & 0,02 & 0,1 & 0,07 & 0,11 & 0,12 & 0,06 & 0,19 & 0,12 \\
\hline Salitre & 0,24 & $0,36^{*}$ & 0,13 & 0,07 & 0,32 & 0,25 & 0,29 & $0,36^{*}$ \\
\hline
\end{tabular}

*Significância estatística da correlação ao nível de 90\%. **Significância estatística da correlação ao nível de 95\%. 
Tabela 3 - Correlação do número de dias consecutivos úmidos (DCU) para a mesorregião Sul Cearense com as anomalias de Temperatura de Superfície do Mar para o período de 1963 a 2014.

\begin{tabular}{lcccccccc}
\hline Estações & SOI & NINO3 & TNA & TSA & ONI & Nino1+2 & Nino 4 & Nino 3,4 \\
\hline Altaneira & $0,31^{* *}$ & $0,27^{* *}$ & $0,27^{* *}$ & 0,13 & $0,23^{*}$ & $0,25^{*}$ & $0,34^{* *}$ & $0,28^{* *}$ \\
Araripe & 0,12 & 0,05 & 0,18 & $0,23^{*}$ & 0,18 & 0,03 & 0,04 & 0,12 \\
Assaré & 0,02 & 0,01 & 0 & 0,21 & 0,02 & 0,09 & 0,13 & 0,04 \\
Aurora & 0,08 & 0,01 & 0,19 & 0,05 & 0,03 & 0,01 & 0,04 & 0 \\
Barbalha & 0,07 & 0,21 & 0,13 & 0,06 & 0,21 & 0,13 & 0,19 & $0,23^{*}$ \\
Brejo santo & 0,15 & 0,03 & $0,38^{* *}$ & 0,21 & 0,06 & 0,04 & 0,01 & 0,03 \\
Brito farias & 0,21 & 0,16 & 0,16 & 0,05 & 0,17 & 0,11 & 0,07 & 0,14 \\
Caririaçu & 0,24 & 0,2 & 0,19 & $0,35^{* *}$ & $0,31^{* *}$ & 0,09 & $0,29 * *$ & $0,28^{* *}$ \\
Crato & 0,08 & 0,03 & 0,03 & 0,18 & 0,11 & 0,04 & 0,07 & 0,07 \\
Granjeiro & 0,17 & 0,06 & 0,21 & 0,21 & 0,12 & 0,05 & 0,02 & 0,07 \\
Jardim & 0,02 & 0,05 & $0,38^{* *}$ & 0 & 0,03 & 0 & 0,13 & 0,07 \\
Mauriti & 0,02 & 0,09 & 0,1 & 0,02 & 0,07 & 0,09 & 0,03 & 0,05 \\
Milagres & $0,22 *$ & 0,04 & $0,37^{* *}$ & 0,1 & 0,14 & 0,02 & 0,02 & 0,08 \\
Missão Velha & 0,18 & 0,05 & $0,40^{* *}$ & $0,24 *$ & 0,08 & 0,05 & 0,03 & 0,05 \\
Porteira & 0,01 & 0,1 & 0,06 & 0,04 & 0,08 & 0,09 & 0,01 & 0,06 \\
Santana & 0,07 & 0,17 & $0,3^{* *}$ & 0,13 & 0,15 & 0,19 & 0,1 & 0,13 \\
Campos Sales & 0,21 & 0 & $0,38^{* *}$ & 0,19 & 0,1 & 0 & 0,03 & 0,03 \\
Barro & 0,24 & 0,16 & 0 & 0,16 & 0,22 & 0,09 & 0,19 & 0,21 \\
Abaia & 0,08 & 0,01 & 0,09 & 0,15 & 0 & 0,09 & 0,09 \\
Penaforte & 0,03 & 0,09 & 0,17 & 0,02 & 0,06 & 0,08 & 0,11 & 0,27 \\
Salitre & 0,19 & 0,26 & 0,25 & 0,11 & 0,26 & 0,12 & $0,30^{*}$ \\
\hline
\end{tabular}

*Significância estatística da correlação ao nível de 90\%. **Significância estatística da correlação ao nível de 95\%.

Tabela 4 - Correlação do índice precipitação total mm/ano (PRCPTOT) para a mesorregião Sul Cearense com as anomalias de Temperatura de Superfície do Mar para o período de 1963 a 2014.

\begin{tabular}{|c|c|c|c|c|c|c|c|c|}
\hline Estações & SOI & NINO 3 & TNA & TSA & ONI & Niño $1+2$ & Niño 4 & Niño 3,4 \\
\hline Altaneira & $0,46^{* *}$ & $0,44 * *$ & 0,2 & $0,24^{*}$ & $0,39 * *$ & $0,38^{* *}$ & $0,35^{* *}$ & $0,41 * *$ \\
\hline Araripe & 0 & 0 & $0,22 *$ & 0,17 & 0,08 & 0,03 & 0,08 & 0,01 \\
\hline Assaré & $0,28 * *$ & $0,38 * *$ & $0,28 * *$ & $0,42 * *$ & $0,28 * *$ & $0,42 * *$ & $0,24 *$ & $0,29 * *$ \\
\hline Aurora & $0,44 * *$ & $0,44 * *$ & 0,13 & 0,2 & $0,43 * *$ & $0,37 * *$ & $0,40^{* *}$ & $0,43 * *$ \\
\hline Barbalha & 0,11 & $0,26^{*}$ & 0,01 & $0,49 * *$ & 0,21 & $0,23^{*}$ & 0,11 & 0,19 \\
\hline Brejo santo & $0,25^{*}$ & $0,29 * *$ & $0,53 * *$ & 0,06 & $0,28 * *$ & 0,2 & $0,38 * *$ & $0,33 * *$ \\
\hline Brito farias & 0,02 & 0,09 & $0,24 *$ & $0,37 * *$ & 0,08 & 0,12 & 0,08 & 0,08 \\
\hline Caririaçu & $0,34 * *$ & $0,45 * *$ & $0,23 *$ & 0,19 & $0,31 * *$ & $0,44 * *$ & $0,35^{* *}$ & $0,36^{* *}$ \\
\hline Crato & $0,27^{*}$ & $0,35 * *$ & 0,13 & $0,41 * *$ & $0,26^{*}$ & $0,37 * *$ & 0,2 & $0,27 * *$ \\
\hline Granjeiro & $0,41 * *$ & $0,52 * *$ & $0,26^{*}$ & 0,13 & $0,42 * *$ & $0,54 * *$ & $0,42 * *$ & $0,46^{* *}$ \\
\hline Jardim & 0,13 & $0,23^{*}$ & $0,31^{* *}$ & 0,01 & 0,12 & $0,22 *$ & 0,2 & 0,17 \\
\hline Mauriti & $0,24^{*}$ & $0,28 * *$ & 0,06 & $0,28 * *$ & 0,21 & $0,27^{*}$ & $0,27 * *$ & $0,24 *$ \\
\hline Milagres & 0,08 & 0,12 & $0,36^{* *}$ & 0,09 & 0 & 0,1 & 0,11 & 0,06 \\
\hline Missão velha & $0,28 * *$ & $0,37 * *$ & $0,36^{* *}$ & 0,13 & $0,33 * *$ & $0,31 * *$ & $0,30 * *$ & $0,35^{* *}$ \\
\hline Porteira & $0,35 * *$ & $0,35 * *$ & $0,33 * *$ & 0,01 & $0,28 * *$ & $0,32 * *$ & $0,37 * *$ & $0,33 * *$ \\
\hline Santana & $0,48 * *$ & $0,43 * *$ & $0,30^{* *}$ & 0,18 & $0,42 * *$ & $0,40 * *$ & $0,37 * *$ & $0,41 * *$ \\
\hline Campos sales & 0,11 & $0,28 * *$ & $0,40 * *$ & 0,07 & 0,17 & $0,24 *$ & $0,28 * *$ & $0,24 *$ \\
\hline Barro & $0,36^{* *}$ & $0,36 * *$ & $0,36^{* *}$ & 0,23 & $0,31 * *$ & $0,30 * *$ & $0,37 * *$ & $0,35^{* *}$ \\
\hline Abaia & $0,40 * *$ & $0,44 * *$ & 0,19 & $0,29 *$ & $0,43 * *$ & $0,29 *$ & $0,38^{* *}$ & $0,43 * *$ \\
\hline Penaforte & $0,37 * *$ & $0,40 * *$ & 0,13 & 0,25 & $0,34 * *$ & $0,31^{*}$ & 0,25 & $0,33 * *$ \\
\hline Salitre & $0,25 * *$ & 0,18 & 0,22 & 0,17 & 0,24 & 0 & 0,31 & 0,28 \\
\hline
\end{tabular}

*Significância estatística da correlação ao nível de 90\%. **Significância estatística da correlação ao nível de 95\%. 
das as frentes frias. Deste modo, quando a precipitação é em escala anual, a resposta é melhor pois se torna mais geral e compreende a sazonalidade dos sistemas atmosféricos oriundos do Atlântico e Pacífico.

\section{Conclusão}

A porção leste da Mesorregião Sul Cearense apresentou importantes quanto a tendência de aumento da aridez. Neste local, verifica-se que o número de dias consecutivos secos aumentou significativamente. Acompanhado disto, verifica-se também redução do volume total de precipitação anual. Este quadro possibilita concluir que a área vem sendo bastante afetada pelos eventos de seca que assolaram o semiárido neste período.

A análise de tendência por meio do teste de Mann-Kendall indica que a maioria dos postos apresentam tendência negativa em suas séries. Porém, poucos apresentaram tendência estatisticamente significante, o que representa de fato mudança no padrão. Estes postos também estão concentrados ao leste da região.

O cruzamento dos dados obtidos por meio de índices com as anomalias de TSM do Atlântico e Pacífico indicam que houve correlação estatisticamente significantes do padrão da precipitação na região e as variações térmicas dos oceanos Pacífico e Atlântico. Para o DCS, as áreas mais influenciadas pelas anomalias de TSM foram as centrais e a leste da Mesorregião Sul Cearense. Este padrão espacial também foi repetido para o número de dias consecutivos úmidos. Para o PCPRTOT, que corresponde ao volume total de precipitação anual, verificou-se que toda a região é influenciada pelas anomalias de TSM do Atlântico e Pacífico.

\section{Referências}

ARAÚJO, W.D.S.; BRITO, J.I.B. DE. Índices de tendências de mudanças climáticas para os estados da Bahia e Sergipe por meio de índices pluviométricos diários e sua relação com TSM do pacífico e atlântico. Revista Brasileira de Meteorologia, v. 26, n. 4, p. 541-554, 2011.

ASSIS, J.M.O.; SOUZA, W.M.; SOBRAL, M.C.M. Climate analysis of the rainfall in the lower-middle stretch of the São Francisco river basin based on the rain anomaly index. Revista Brasileira de Ciências Ambientais (Online), v. 2, p. 188-202, 2015.

BATES, B.C.W.K.; ZBIGNIEW, S.; WU, E.; PALUTIKOF, J.P. El Cambio Climático y el água. Secretaría de IPCC: Ginebra, 2008.

CAMPOS, N.A. A grande seca de 1979 a 1983: um estudo de caso das ações do Governo em duas subregiões do Estado do Ceará. Teoria e Pesquisa. UFSCar. São Paulo, 2004.

GALLANT, A.; KAROLY, D.; GLEASON, K. Consistent trends in modified climate extremes index in the U.S.A, Europe and Australia. Journal of Climate, v. 27, p. 1379-1394, 2014.

GOOSSENS, C.; BERGER, A. Annual and seasonal climatic variations over the northern hemisphere and Europe during the last century. Annales Geophysicae, Berlin, v. 4, n.4, p. $385-400,1986$.

FALLAH, B.; CUBASCH, U.; PRÖMMEL, K; SODOUDI, S. A numerical model study on the behaviour of Asian summer monsoon and AMOC due to orographic forcing of Tibetan Plateau. Climate Dynamics, v. 47, n. 1485. 2016.

HAYLOCK, M.; PETERSON, T.; ALVES, L.; AMBRIZZI, T.; ANUNCIAÇÃO, M. et al. Trends in total and extreme South American rainfall 1960-2000 and links with sea surface temperature. Journal of Climate, v. 19, p. 1490-1512, 2006.

HASTENRATH, S.; HELLER, L. Dynamics of climatic hazards in Northeast Brazil. Quartely Journal Royal Meteorological Society, v. 103, n. 435, p. 77-92, 1977.

IPCC. Climate Change 2013: The Physical Science Basis. Contribution of Working Group I to the Fifth Assessment Report of the Intergovernmental Panel on Climate Change [Stocker, T.F., D. Qin, G.-K. Plattner, M. Tignor, S.K. Allen, J. Boschung, A. Nauels, Y. Xia, V. Bex and P.M. Midgley (eds.)]. Cambridge University Press, Cambridge, United Kingdom and New York, NY, USA, 2013. 1535p.

LUCE C.H. Effects of climate change on snowpack, glaciers, and water resources in the Northern Rockies. In: Halofsky J., Peterson D. (eds) Climate Change and Rocky Mountain Ecosystems. Advances in Global Change Research, v. 63, 2018.

MARENGO, J.A. Mudanças climáticas globais e seus efeitos sobre a biodiversidade - caracterização do clima atual e definição das alterações climáticas para o território brasileiro ao longo do século XXI. 2. ed. Brasília: Ministério do Meio Ambiente, 2007. v.1, p.214.

MARENGO J.A.; CUNHA, A.P.; ALVES, L.M. A seca de 201215 no semiárido do Nordeste do Brasil no contexto histórico. Revista Climanálise, v. 3, p. 49-54, 2016.

NÓBREGA, R.S.; SANTIAGO, G.A.C.F. Tendência de temperatura na superfície do mar nos oceanos atlântico e pacífico e variabilidade de precipitação em Pernambuco. Mercator, v. 13, n. 1, p. 107-118, 2014.

NÓBREGA, R.S.; SANTIAGO, G.A.C.F.; SOARES, D.B. Tendência do controle climático oceânico sob a variabilidade temporal da precipitação no Nordeste do Brasil. Revista Brasileira de Climatologia, v. 18, p. 276-292, 2016.

SALVADOR, M. Análise da variabilidade climática na nova fronteira agrícola do Brasil: região do Matopiba. Campina Grande: Universidade Federal de Campina Grande (UFCG), 2014. 119. Tese de doutorado, Programa de pósgraduação em meteorologia, Campina Grande.

SANTOS, C.; BRITO, J.; RAO, T.; MENEZES, H. Tendências dos índices de precipitação no estado do Ceará. Revista Brasileira de Meteorologia, v. 24, p. 39-47, 2009.

SANTOS, P.V; SANTOS, R.; COUTINHO, M.D.L. Detecção de Mudanças Climáticas Através de Índices Pluviométricos Diários no Estado de Pernambuco. Revista Brasileira de Geografia Física, v. 6, n. 4, p. 713-731, 2013.

SKANSI, M.; BRUNET, M.; SIGRÓ, J.; AGUILAR, E.; GROENING, J.; BENTANCUR, O. et al. Warming and wetting signals emerging from analysis of changes in climate extreme indices over South America. Global and Planetary Change, v. 100, p. 295-307, 2013.

SOUZA, W.M.; AZEVEDO, P.V. Índices de detecção de Mudanças Climáticas Derivados da Precipitação Pluviométrica e 
das Temperaturas em Recife-PE. Revista Brasileira de Geografia Física, v. 06, n. 1, p. 143-159, 2012.

SNEYERS, R. Sur I'analyse statistique des séries d'observations. Genève: Organisation Météorologique Mondial, 1975. 192 p. (OMM Note Technique, 143).

TABARI, H.; TALAEE, P.H. Temporal variability of precipitation over Iran: 1966-2005. Journal of Hydrology, v. 396, n. 3-4, p. 313-320, 2011.

TETTEY, M.; ODURO, F.; ADEDIA, D.; ABAYE, D. Markov chain analysis of the rainfall patterns of five geographical locations in the south eastern coast of Ghana. Earth Perspectives, v.4, n.6, 2017.

VENETSANOU, P.; KAZAKIS, N.; ANAGNOSTOPOULOU, C.; VOUDORIS, K. Impacts of rainfall changes on groundwater balance of coastal aquifers: a case study of the Thermaikos Gulf, North Greece. Global NEST Journal, v. 18, (In progress), 2016.

\section{Endereços de Internet}

IBGE - INSTITUTO BRASILEIRO DE GEOGRAFIA E ESTATÍSTICA. Censo 2010. Disponível em: http://www.censo2010.ibge.gov.br/sinopse. Acesso em: 20 agos. 2017.

NOAA - NATIONAL OCEANIC AND ATMOSPHERIC ADMINISTRATION. Disponível em: http://www.esrl.noaa.gov/psd/data/climateindices/list. Acesso em 24 abr. 2017.

UFCG - UNIVERSIDADE FEDERAL DE CAMPINA GRANDE. Climap. Disponível em: http://www.dca.ufcg.edu.br/. Acesso em 20 jun. 2017.

This is an Open Access article distributed under the terms of the Creative Commons Attribution Non-Commercial License which permits unrestricted non-commercial use, distribution, and reproduction in any medium provided the original work is properly cited. 